\title{
Prevalencia y factores asociados al deseo sexual hipoactivo en hombres del Quindío
}

\section{Prevalence and Factors Associated with Hypoactive Sexual Desire in Quindío Men}

\author{
Franklin José Espitia De La Hoz 1,2,30 \\ ${ }^{1}$ Ginecología y Obstetricia, Universidad Militar nueva Granada, \\ Bogota, Columbia \\ 2 Sexología Clínica, Universidad de Alcalá de Henares, Madrid, Spain \\ 3 Uroginecología / FUCS, Hospital de San José / Unicamp, Brasil
}

\author{
Address for correspondence Franklin José Espitia De La Hoz, Carrera \\ 12 \# 0-75, Consultorio 508, Clínica del Café, Armenia, Quindío, \\ Colombia (e-mail: espitiafranklin@hotmail.com).
}

\section{Resumen}

Palabras clave

- disfunciones sexuales psicológicas

- hombres

- prevalencia

- factores de riesgo

- sexualidad
Introducción El deseo sexual hipoactivo describe el bajo interés hacia la actividad sexual en general, caracterizando la escasa o nula motivación para tener relaciones eróticas, con disminución o ausencia de pensamientos o fantasías sexuales.

Objetivo Evaluar la prevalencia y factores asociados, al deseo sexual hipoactivo en hombres del Quindío, así como estimar las demás disfunciones sexuales.

Métodos Estudio observacional. La población estuvo constituida por 171 hombres que asistieron a consulta externa en una clínica universitaria de la ciudad de Armenia, Colombia, en el 2019. Se excluyeron los hombres menores de 18 años, residentes fuera del Quindío, situación psicopatológica o social que dificultara la comprensión del instrumento y los que no consintieron participar en el estudio. Se aplicó como instrumento el "Massachusetts General Hospital-Sexual Functioning Questionnaire (MGH-SFQ)". Se evaluaron las características sociodemográficas, estilos de vida, salud sexual y reproductiva, antecedentes y comportamiento sexual. Se hizo análisis descriptivo.

Resultados La edad promedio fue de $41,79 \pm 11,46$ años (rango 18-81). La prevalencia de disfunciones sexuales en el grupo estudiado fue de $21,63 \%$. La puntuación del MGH-SFQ fue de 14,61 $\pm 4,23$ puntos (variación: 7,26-19,26). Se presentaron dificultades con el interés sexual (15,78\%), excitación sexual $(6,43 \%)$, orgasmo (8,77\%), erección ( $21,63 \%$ y yatisfacción sexual global $(12,28 \%)$. La mediana de disfunciones sexuales por hombre fue de 2 , que se hizo presente en el $27,48 \%$ \% El análisis multivariado (regresión logística) mostró que los factores asociados al deseo sexual hipoactivo fueron testosterona baja (OR: 5,59; IC95\% 1,82-18,37), ansiedad / depresión (OR: 5,53; IC95\% 1,72-18,43), convivencia en pareja mayor a 10 años (OR: 5,19; IC95\%: 2,71-11,71), ansiedad de desempeño (OR: 4,62; IC95\% 1,95-10,56), incremento de la edad (OR: 3,42; IC95\%: 1,26-9,36), cansancio / estrés (OR: 2,58; IC95\%: 1,08-3,28), trastornos del sueño (OR: 1,89; IC95\%: 1,35-2,58), conflictos de pareja (OR: 1,53; IC95\%: 1,02-2,37) y antecedente de disfunciones sexuales (OR: 1,47; IC95\%: 0,99-2,22); received

January 14, 2020

accepted

June 17, 2020

published online

October 6, 2020
DOI https://doi.org/

$10.1055 / \mathrm{s}-0040-1714724$.

ISSN 0120-789X.

e ISSN 2027-0119. (c) 2020. Sociedad Colombiana de Urología. All rights reserved. This is an open access article published by Thieme under the terms of the Creative Commons Attribution-NonDerivative-NonCommercial-License, permitting copying and reproduction so long as the original work is given appropriate credit. Contents may not be used for commercial purposes, or adapted, remixed, transformed or built upon. (https://creativecommons.org/ licenses/by-nc-nd/4.0/)

Thieme Revinter Publicações Ltda., Rua do Matoso 170, Rio de Janeiro, RJ, CEP 20270-135, Brazil 


\section{Abstract}

\section{Keywords}

- sexual dysfunctions

- psychological

- men

- prevalence

- risk factors

- sexuality mientras que, el uso de juguetes sexuales (OR: 0,78 ; IC95\%: 0,72-0,96; $p=0,021$ ), consumo de vitamina D (2000 UI / diarias) (OR: 0,64; IC95\%: 0,42-0,96) o de Inhibidores de fosfodiesterasa-5 (OR: 0,78; IC95\%: 0,63-0,93) constituyeron factores protectores.

Conclusiones En el presente estudio, el 21,63\% de los hombres presentaron disfunciones sexuales. Los trastornos de la erección $(21,63 \%$ ) y el interés sexual (15,78\%), fueron los más afectados. La testosterona baja, ansiedad / depresión y convivencia en pareja mayor a 10 años, encabezan los principales factores asociados al deseo sexual hipoactivo. El hacer actividades juntos (OR: 0,44; IC95\%: 0,34-0,68), el respeto a ser personas diferentes (OR: 0,53; IC95\%: 0,41-0,71), mantener la armonía en la pareja (OR: 0,61; IC95\%: 0,47-0,79) y la expresión de sentimientos a la pareja (OR: 0,68; IC95\%: 0,46-0,95) constituyen una línea de protección para mejorar las estrategias de prevención de los trastornos sexuales en esa población.

Introduction Hypoactive sexual desire describes the low interest in sexual activity in general, characterizing the little or no motivation to have erotic relationships, with a decrease or absence of sexual thoughts or fantasies.

Objective To determine the sexual dysfunctions and to evaluate the prevalence and associated factors, to the hypoactive sexual desire in men of Quindío.

Methods Observational study. The population consisted of 171 men who attended an outpatient clinic at a university clinic in the city of Armenia, Colombia, in 2019. Men under 18 years of age, residents outside of Quindío, psychopathological or social situation that made understanding difficult, were excluded of the instrument and those who did not consent to participate in the study. The "Massachusetts General Hospital-Sexual Functioning Questionnaire (MGH-SFQ)" was applied as an instrument. Socio-demographic characteristics, lifestyles, sexual and reproductive health, background and sexual behavior were evaluated. Descriptive analysis was done.

Results The average age was $41.79 \pm 11.46$ years (variation: -81 ). The prevalence of sexual dysfunctions in the study group was $21.63 \%$. The MGH-SFQ score was $14.61 \pm 4.23$ points (range between 7.26 - 19.26). There were difficulties with sexual interest $(15.78 \%)$, sexual arousal $(6.43 \%)$, orgasm $(8.77 \%)$, erection $(21.63 \%$ ) and overall sexual satisfaction (12.28\%). The median sexual dysfunction per man was 2 , which was present in $27.48 \%$. The multivariate analysis (logistic regression) showed that the factors associated with hypoactive sexual desire were low testosterone (OR: 5.59; $95 \% \mathrm{Cl} 1.82-18.37$ ), anxiety / depression (OR: $5.53 ; 95 \% \mathrm{Cl} 1.72-18.43$ ), cohabitation in a couple older than 10 years (OR: 5.19; 95\% Cl: 2.71-11.71), performance anxiety (OR: 4.62; 95\% Cl 1.95-10.56), increase in age (OR: 3.42; 95\% Cl: 1.26-9.36), fatigue / stress (OR: 2.58; 95\% Cl: 1.08-3, 28), sleep disorders (OR: 1.89; 95\% Cl: 1.35-2.58), couple conflicts (OR: 1.53; 95\% Cl: 1.02-2.37) and a history of sexual dysfunctions (OR: 1.47 ; $95 \% \mathrm{Cl}: 0.99-2.22$ ); while, the use of sex toys (OR: $0.78 ; 95 \%$ Cl: $0.72-0.96 ; p=0.021$ ), vitamin D consumption (2000 IU / daily) (OR: 0.64; 95\% Cl: $0.42-0.96)$ or of phosphodiesterase-5 inhibitors (OR: 0.78 ; $95 \%$ Cl: $0.63-0.93$ ) constituted protective factors.

Conclusions In the present study, $21.63 \%$ of men had sexual dysfunction. Disorders of erection $(21.63 \%)$ and sexual interest $(15.78 \%)$ were the most affected. Low testosterone, anxiety / depression and coexistence in a couple older than 10 years, lead the main factors associated with hypoactive sexual desire. Low testosterone, anxiety / depression and coexistence in couples older than 10 years, are the main factors associated with hypoactive sexual desire. Doing activities together (OR: 0.44, 95\% Cl: 0.34-0.68), respect for being different people (OR: 0.53, 95\% Cl: 0.41-0.71), maintaining harmony in the couple (OR: $0.61 ; 95 \% \mathrm{Cl}: 0.47-0.79)$ and the expression of feelings toward the couple (OR: $0.68 ; 95 \% \mathrm{Cl}: 0.46-0.95)$ constitute a protection line to improve prevention strategies for sexual disorders in this population. 


\section{Introducción}

La función sexual en una persona, es adecuada cuando existe una óptima interrelación entre los factores psicológicos, emocionales, neurológicos, vasculares y endocrinos, ${ }^{1,2}$ y naturalmente bajo el influjo de la conveniente armonía con su vínculo o pareja. ${ }^{3}$

Las disfunciones sexuales, presentes en orden de aparición en los hombres, son: eyaculación precoz (afecta alrededor del $30 \%$ de la población, considerándose el trastorno sexual más común entre los varones); seguido por la disfunción eréctil (alcanza hasta un 20\% de los hombres mayores de 40 años, con una incidencia cuatro veces superior en los mayores de 70 años -en comparación con los hombres menores de 20 años-). 2,3 La prevalencia de bajo deseo sexual en hombres de 16 a 59 años varía entre el $14 \%$ y $17 \%$, con un aumento de la prevalencia a edades más avanzadas. ${ }^{4,5}$

El deseo sexual es una construcción compleja, ${ }^{6}$ por lo tanto, ha sido definido de manera diversa. Se ha conceptualizado como sensaciones que motivan a los individuos a iniciar o ser receptivos a la estimulación sexual. ${ }^{7}$ Por otro lado, Regan y Berscheid, ${ }^{8}$ lo definen como "un estado psicológico experimentado subjetivamente por el individuo como una conciencia de que él o ella quiere o desea alcanzar un objetivo sexual (presumiblemente placentero) que actualmente es inalcanzable."

Es usual una disminución temporal del interés sexual, la cual suele estar asociada a inductores temporales como el cansancio físico o el estrés; no obstante, el deseo sexual hipoactivo se convierte en un trastorno o disfunción, cuando los pensamientos sexuales, las fantasías y el deseo de actividad sexual disminuyen durante un periodo prolongado de tiempo, más de lo que debería esperarse de acuerdo a la edad y duración de la relación de pareja; el cual a su vez, causa angustia o se encuentra presente a todo lo largo de la experiencia o encuentro sexual. ${ }^{9}$

La presencia de alteraciones emocionales y dificultades en la relación de pareja, pueden contribuir a una vida sexual menos activa y por ende menos satisfactoria. ${ }^{9}$ El envejecimiento, la pérdida de la autoestima, disfunciones sexuales, disminución de la satisfacción sexual, dolor durante las relaciones sexuales, falta de comunicación y comprensión por parte de la pareja, etc., pueden afectar el interés o deseo sexual. ${ }^{10}$

El estudio de las disfunciones sexuales en hombres, ha sido una tarea titánica en Colombia, dado que han surgido todo tipo de limitaciones; por un lado está el tema de la cobertura y por el otro, se encuentra el tipo de población, no siendo fácil enrolar poblaciones poco heterogéneas, donde no sean amplios los sesgos de selección ${ }^{11}$; es por eso que el objetivo de esta investigación, consiste en estimar la prevalencia de las disfunciones sexuales, y caracterizar los factores asociados al deseo sexual hipoactivo en hombres del Quindío.

\section{Métodos}

Diseño y población. Estudio observacional descriptivo de corte transversal, en el que se incluyeron hombres que asistieron a la consulta externa en una clínica universitaria, de mediana complejidad en la ciudad de Armenia; institución de naturaleza privada, que atiende a población afiliada al régimen de aseguramiento subsidiado y contributivo por el Estado en el sistema de seguridad social en Colombia; en el periodo del 01 de febrero al 31 de agosto de 2019. Se excluyeron los hombres menores de 18 años, residentes fuera del Quindío, situación psicopatológica o social que dificultara la comprensión del instrumento y los que no consintieron participar en el estudio. Se utilizó como instrumento el "Massachusetts General Hospital-Sexual Functioning Questionnaire (MGH-SFQ)". El MGH-SFQ es un cuestionario auto administrado, diseñado para detectar problemas con la función sexual y ha sido validado en español. ${ }^{12,13}$ Es un cuestionario conformado por 5 ítems: interés, excitación, orgasmo, erección y satisfacción global. La respuesta se mide según el siguiente puntaje: 0) Totalmente disminuido; 1) Marcadamente disminuido; 2) Moderadamente disminuido; 3) Mínimamente disminuido; 4) Normal. Las puntuaciones altas indican un mejor funcionamiento sexual, y las menores de 4 (en al menos un elemento del cuestionario), se consideran como disfunción. El rango total de la puntuación del MGH-SFQ va desde 0 hasta 20 (-Anexo 1); menores puntuaciones indican peor funcionamiento sexual, por lo tanto, se considera riesgo de disfunción sexual cuando al menos un elemento arroja una puntuación inferior a 4 o en la puntación total. La versión adaptada al español presenta una excelente fiabilidad de consistencia interna, 0,90 en hombres, así como adecuados indices de validez. ${ }^{12,13}$

Se tomó como variable dependiente la presencia de deseo sexual hipoactivo, y como variables independientes: edad, antecedentes patológicos y quirúrgicos, consumo de medicamentos, hábitos, cansancio / estrés, relación de pareja, niveles de testosterona $y$ salud sexual $y$ reproductiva. Se conformaron dos grupos definidos por la presencia o ausencia de deseo sexual hipoactivo, los que se utilizaron para la elaboración de tablas de dos por dos con cada uno de los factores asociados.

El tamaño de la muestra se estimó teniendo como referencia el promedio de hombres atendidos en la consulta externa, en el periodo correspondiente al semestre, inmediatamente anterior al diseño del estudio (01 de agosto de 2018 a 31 de enero de 2019), con un error aleatorio inferior al 5\%, una proporción esperada de adherencia del 50\% y un nivel de confianza del 95\%; teniendo en cuenta el factor por población finita, y así, la muestra seleccionada fue de 159 hombres. Se hizo un muestreo aleatorio simple mediante una tabla de números aleatorios. El cálculo se realizó mediante el programa estadístico Epidat 3.1.

\section{Procedimiento}

En la institución participante, los hombres que acudieron a la consulta externa, fueron evaluados por un médico familiar, diplomado en Sexología Clínica, a fin de determinar si cumplían con los criterios de elegibilidad del estudio. Si el hombre cumplía con los criterios, se le informaban los objetivos de la investigación, así como el propósito que 
tenían los resultados, garantizándoles la confidencialidad de la información, y solicitándoles la firma del consentimiento informado. Una vez obtenido el consentimiento, dos enfermeras profesionales (encargadas de la recolección del instrumento estandarizado), le daban instrucciones para el diligenciamiento del cuestionario "Massachusetts General Hospital-Sexual Functioning Questionnaire (MGH-SFQ)" a cada uno de los hombres, de manera individual y en un ambiente privado.

La determinación cuantitativa de la Testosterona Total se hizo mediante un método validado por LC-MSMS (cromatografía líquida en tándem con espectrometría de masa); siguiendo el Consenso del Centers for Disease Control and Prevention (CDC)'s Hormone Standardization Project (CDCHoST Program). Se recolectó sangre venosa periférica por la mañana, y a todos los participantes se les hizo dos mediciones en ocasiones separadas. Se utilizó un nivel de testosterona total por debajo de $300 \mathrm{ng} / \mathrm{dL}$, como punto de corte para definir los niveles bajos de testosterona.

El equipo de investigación estuvo conformado por el investigador principal, el cual realizó la atención de cada participante, dos enfermeras profesionales y un médico familiar (diplomado en Sexología Clínica), así como por tres auxiliares de enfermería entrenadas en la recolección de datos, las cuales a su vez eran coordinadas por una enfermera profesional, diplomada en Métodos estadísticos para el análisis de datos; todas eran expertas en el diligenciamiento del "Massachusetts General Hospital-Sexual Functioning Questionnaire (MGH-SFQ)" y conocedoras, por completo, de los intereses de la investigación.

Variables medidas. Socio-demográficas (edad, raza, nivel de estudios, nivel socio-económico, estado civil, ocupación, afiliación al sistema general de seguridad social en salud, tiempo de convivencia en pareja, condición espiritual o religiosa, área de residencia); estilos de vida (índice de masa corporal, horas de sueño, consumo de tabaco, ingesta de alcohol, ejercicio físico, uso de sustancias psicoactivas). Antecedentes patológicos personales y familiares, antecedentes quirúrgicos; variables de salud sexual y reproductiva (edad de inicio de vida sexual, número de hijos, uso del preservativo, antecedente personal de disfunción sexual e infecciones de transmisión sexual / ITS); variables de comportamiento sexual (orientación sexual, recurrencia a la masturbación, prácticas sexuales penetrativas, prácticas buco - genitales, frecuencia promedio de relaciones sexuales semanales, frecuencia para lograr alcanzar el orgasmo, número de parejas sexuales, tiempo de convivencia en pareja, antecedente de abuso sexual o violencia sexual en el matrimonio, pareja con disfunción sexual, uso de medicamentos e infidelidad). Se indagaron además las preguntas del instrumento "Massachusetts General Hospital-Sexual Functioning Questionnaire (MGH-SFQ)"; adicionalmente, se consideró el análisis por subgrupos de edad (18-29, 30-39, 40-49, 50-59, 60-69, mayores de 70 años) para hacer una comparación final del comportamiento porcentual de la prevalencia de disfunciones sexuales en los hombres en esos rangos de edad.

Análisis estadístico. La información fue llevada a una base de datos en Microsoft Office Excel 2007 (v12.0). El análisis de la información se hizo con el paquete estadístico Stata versión 14. Las variables cuantitativas se expresaron a través de medidas de tendencia central con su respectiva medida de dispersión (rango intercuartil para mediana y desviación estándar para promedios). Las variables categóricas se expresaron en frecuencias y proporciones y fueron comparadas usando la prueba de $x^{2}$ de Pearson o prueba Exacta de Fisher. Algunas variables se agruparon para su análisis. Para determinar la asociación entre cada variable independiente y la variable resultado, se calculó el Odds Ratio (OR) con su intervalo de confianza del 95\% (IC95\%). Se hizo el análisis de las variables y la determinación de las asociaciones. El análisis multivariado se realizó mediante regresión logística. Se consideró un valor de $p<0,05$ como estadísticamente significativo.

Aspectos éticos. La investigación fue aprobada por el comité de ética de la institución de salud participante con número HCS0001-003.

\section{Resultados}

El promedio de edad fue de $41,79 \pm 11,46$ años, mediana de 37 años (rango 18-81), superior a la de sus parejas (37,56 $\pm 10,83$ años; mediana de 32 años, rango 15-75). Se observó una heterogénea distribución etárea, distribuida así: $8,77 \%$ tenía entre 18 y 29 años; $12,28 \%$ entre 29 y 39 años; $24,56 \%$ entre 40 y 49 años; $19,29 \%$ entre 50 y 59 años; $21,05 \%$ entre 60 y 69 años; y 14,03\% mayores de 70 años. En cuanto a la raza, la muestra estuvo constituida mayoritariamente por hispanos $(68,42 \% / n=117)$. En relación con el estrato socioeconómico, el $63,15 \%(n=108 / 171)$, eran de estrato medio. El $14,61 \%(n=25 / 171)$ no habían terminado la secundaria, pero el $43,85 \%(n=75 / 171)$ eran profesionales. El $89,47 \%$ $(n=153 / 171)$ pertenecían al régimen contributivo de seguridad social en salud. El 85,96\% $(n=147 / 171)$ profesaban la religión católica, y el $78,94 \%(n=135 / 171)$ residían en el área urbana. En la - Tabla 1 se describen las características socio-demográficas.

La prevalencia de enfermedades crónicas no transmisibles se detalla en la - Tabla 2. La ingesta de medicamentos reportó una mediana de 3 fármacos por participante (rango entre 0-12). El 14,03\% presentaban antecedente personal de depresión, frente al 21,05\% de antecedente familiar.

El 32,16\% reportaron perturbación relacionadas con el sueño, donde el $12,72 \%$ habían recibido tratamiento por insomnio. El 80,11\% manifestó dormir menos de 8 horas, siendo del $92,59 \%$ entre los profesionales de la salud que hacen turnos nocturnos, relación estadísticamente significativa $(p=0,0001)$. El 93,56\% consumían alcohol por lo menos una vez en la semana. El tabaquismo alcanzó el $23,39 \%$, y el consumo de sustancias psicoactivas el $5,26 \%$. El $68,42 \%$ se ejercitaba de forma regular ( $\geq 3$ veces a la semana).

El 57,89\% tenían antecedentes quirúrgicos; en orden de frecuencia: colecistectomía (27,27\%), safenectomía (21,21\%), apendicectomía (18,18\%), plastías inguinales con colocación de malla $(15,15 \%)$, laparotomías exploradoras $(12,12 \%)$, vasectomía $(11,69 \%)$, plastías umbilicales $(9,09 \%)$, resección 
Tabla 1 Características socio-demográficas de los hombres con deseo sexual hipoactivo en el Quindío, $2019(n=171)$

\begin{tabular}{|l|l|}
\hline Variable y categorías & $\mathbf{n} /$ proporción (\%) \\
\hline Edad: $X \pm D E$ años & $41,79 \pm 11,46$ \\
\hline Edad de la pareja: $X \pm \mathrm{DE}$ & $37,56 \pm 10,83$ \\
\hline Peso: $\mathrm{X} \pm \mathrm{DE} \mathrm{Kg}$ & $76,59 \pm 9,33$ \\
\hline Talla: $\mathrm{X} \pm \mathrm{DE}$ Cms & $167,49 \pm 5,73$ \\
\hline IMC: $\mathrm{X} \pm \mathrm{DE}$ & $27,33 \pm 7,26$ \\
\hline $\begin{array}{l}\text { Tiempo de convivencia } \\
\text { en pareja: } \mathrm{X} \pm \text { DE años }\end{array}$ & $13,59 \pm 4,56$ \\
\hline Estado civil & \\
\hline Casados & $47(27,48 \%)$ \\
\hline Unión libre & $67(39,18 \%)$ \\
\hline Solteros & $16(9,35 \%)$ \\
\hline Divorciados & $41(23,97 \%)$ \\
\hline Ocupación & $91(53,21 \%)$ \\
\hline Empleados & $53(30,99 \%)$ \\
\hline Trabajadores independientes & $8(4,67 \%)$ \\
\hline Desempleados & $19(11,11 \%)$ \\
\hline Pensionados &
\end{tabular}

Tabla 2 Prevalencia de enfermedades crónicas en hombres con deseo sexual hipoactivo en el Quindío, $2019(n=171)$

\begin{tabular}{|l|l|}
\hline Patología & Prevalencia \\
\hline Artritis reumatoide & $2,92 \%$ \\
\hline Artrosis & $6,43 \%$ \\
\hline Cáncer & $21,63 \%$ \\
\hline Diabetes & $10,52 \%$ \\
\hline Dislipidemia & $14,03 \%$ \\
\hline Enfermedad cardiovascular & $24,56 \%$ \\
\hline Enfermedad de Parkinson & $2,33 \%$ \\
\hline Hipotiroidismo & $9,35 \%$ \\
\hline HTA & $27,48 \%$ \\
\hline Prostatismo & $22,22 \%$ \\
\hline Neumopatías crónicas & $5,84 \%$ \\
\hline Osteoporosis & $8,18 \%$ \\
\hline
\end{tabular}

transuretral de próstata para adenoma (7,07\%), funduplicaturas $(5,05 \%)$ y cirugía de corazón abierto (3,03\%); con una mediana de 3 cirugías por participante mayor de 50 años (rango ente 0-9).

En los antecedentes de salud sexual y reproductiva se encontró que la edad promedio de la primera relación sexual fue a $\operatorname{los} 15,37 \pm 1,49$ años, con una mediana de 14 años, (rango 12-21); se detectó que solo el 5,26\% utilizó preservativo en el primer coito. Se obtuvo una mediana de 3 hijos (rango entre 0 y 15); de ellos, el 75,43\% fueron no planeados. El uso sistemático del condón, por parte de los hombres solteros, se encontró en el $26,31 \%$. El $17,54 \%$ declararon padecer alguna disfunción sexual en el último semestre, mientras que el 38,59\% afirmó que la pareja presentaba alguna disfunción sexual. El antecedente de infecciones de transmisión sexual fue del 7,01\%.

En relación con el comportamiento sexual, el 92,98\% se caracterizaron por ser heterosexuales; observándose diversidad en las prácticas sexuales penetrativas: pene vagina (100\%); pene - boca $(77,19 \%)$ y pene - ano $(29,82 \%)$, con un $64,91 \%$ de prácticas buco - genitales. La masturbación es una práctica poco común en el $82,45 \%$ de los hombres encuestados, siendo habitual entre los homosexuales (91,66\%), lo cual resultó estadísticamente significativo $(p<0,001)$. En relación con el número de parejas sexuales se reportó una mediana de 15 (rango entre $1 \mathrm{y} \geq 30$ ). El 45,61\% refirió más de 10 años de tiempo de convivencia en pareja. El 10,52\% afirmó que la pareja le había sido infiel, mientras que el $73,68 \%$ expresó haber sido infiel, por lo menos en una ocasión; y el 4,76\% aceptaron llevar una relación paralela $\geq$ a 3 años.

A la pregunta ¿cuántas veces (a la fecha del estudio), tuvo relaciones sexuales la semana pasada? (periodo transcurrido en los siete días anteriores a la entrevista), el 16,37\% del total de la población manifestó más de cinco veces; de las cuales, el $30,4 \%$ lo hizo en una sola ocasión por encuentro. El 25,14\% lo hacen tres veces a la semana, el $32,74 \%$ lo hace dos veces a la semana, y el $25,73 \%$ lo hace menos de una vez a la semana, arrojando una mediana de 3 encuentros sexuales semanales (rango 0-9). Siendo inferior a 2 (rango 0-4) encuentros sexuales semanales entre los mayores de 50 años. El $13,45 \%$ reportó dificultad para lograr el orgasmo con frecuencia ( $2 / 3$ de las veces).

El antecedente de abuso sexual se encontró en el 6,43\%. La edad promedio al momento del abuso fue de 10,57 $\pm 2,73$ años, con una mediana de 9 años (rango 6-15); en el 81,81\% de los casos la persona abusadora era una mujer, lo cual dificultó el reconocimiento de haber sido abusados. La violencia sexual infligida por la pareja se encontró en el $9,94 \%$. El 18,12\% notificaron que, a consecuencia de su bajo deseo sexual, habían sufrido algún acto de maltrato emocional perpetrado por la pareja. El 94,73\% de los participantes informó haber probado algún tipo de fármaco estimulador sexual (sin prescripción médica), con predominio de los inhibidores de la fosfodiesterasa-5 en el $90,74 \%$.

La enfermedad cardiovascular apareció con una frecuencia significativamente mayor entre los hombres que tuvieron menos de tres encuentros sexuales a la semana (32,14\% frente al 16,78\%; $p=0,01)$.

Los hombres que consumen alcohol (más de tres veces a la semana), mostraron tres veces más probabilidad de presentar deseo sexual hipoactivo, que los no consumidores (OR: 3,29; IC95\%: 2,07-5,19); $p=0,000$ ), mientras que los fumadores tienen casi 3 veces más riesgo frente a los que no fuman (OR: 2,95; IC95\%: 1,59-5,78). La adicción a sustancias psicoactivas aumentó cuatro veces el riesgo (OR: 4,31; IC95\%: 3,04-6,13).

El deseo sexual hipoactivo fue significativamente mayor en los hombres que consumían antidepresivos (62,5\% frente 
Tabla 3 Massachusetts General Hospital-Sexual Functioning Questionnaire (MGH-SFQ) en hombres con deseo sexual hipoactivo en el Quindío, 2019

\begin{tabular}{|l|l|l|}
\hline Dominios & Media $( \pm \mathrm{DE})$ & $\begin{array}{l}\text { Disfunción } \\
\text { sexual (\%) }\end{array}$ \\
\hline Interés sexual & $2,82 \pm 0,81$ & 15,78 \\
\hline Excitación sexual & $3,15 \pm 0,96$ & 6,43 \\
\hline Orgasmo & $3,24 \pm 0,93$ & 8,77 \\
\hline Erección & $2,46 \pm 0,72$ & 21,63 \\
\hline $\begin{array}{l}\text { Satisfacción } \\
\text { sexual global }\end{array}$ & $2,94 \pm 0,87$ & 12,28 \\
\hline Puntaje total & $\begin{array}{l}14,61 \pm 4,23 \\
\text { puntos }\end{array}$ \\
\hline
\end{tabular}

al $44,89 \%$ de los no consumidores $(p=0,001$ ) (OR: 3,65 ; IC95\%: $2,49-4,87) ; p=0,000$ ); siendo la asociación más alta en los que ingieren fármacos por patologías crónicas, con un OR de 4,27 (IC95\%: 3,05-5,27).

Los hombres que refirieron menos problemas con el interés sexual, tenían una mayor tasa de infidelidad en relación con los que padecían más ese trastorno; se observó que el riesgo de deseo sexual hipoactivo es 28 veces mayor en quienes no son infieles, comparado con quienes son infieles frecuentemente (OR: 28,53; IC95\%; 8,79-90,33; $\mathrm{p} \leq 0,000$ ); mientras que aquellos cuyas parejas les habían sido infiel, tuvieron una prevalencia dos veces mayor de deseo sexual hipoactivo (OR: 2,13; IC95\%: 1,64-2,83), frente a los que negaron que sus parejas les fueran infieles.

La puntuación del MGH-SFQ en la población global del total de los 171 hombres, fue de 14,61 $\pm 4,23$ puntos, con una mediana de 13 puntos (rango entre 7- 19). La prevalencia de disfunción sexual fue del 21,63\% en el grupo estudiado. La disfunción sexual presente con más frecuencia fue el trastorno de la erección (21,63\%), seguido por la alteración del interés sexual (15,78\%). En la - Tabla 3 se describe la puntuación detallada de los resultados del MGH-SFQ. El 27,48\% presentó dos disfunciones sexuales, y el 12,86\% 3 o más disfunciones sexuales, con una mediana de disfunciones sexuales por hombre de 2 (rango entre 1 y 4 ), se observó que en los hombres cuya puntuación en el dominio Interés sexual
(MGH-SFQ) fue menor a 2,82 puntos, presentaron 12 veces más riesgo de alteración con la erección (OR: 12,63; IC95\%; 2,49-59,49; $\mathrm{p} \leq 0,001)$ que aquellos cuya puntuación fue mayor a 2,82 puntos.

Se encontró una frecuencia de simuladores del orgasmo del $23,39 \%$, pero solo el 3,5\% refirió saber diferenciar el orgasmo de la eyaculación. El 7,01\% afirmó haber asistido a una consulta de sexología o consejería sexual, de los cuales, únicamente el $2,33 \%$ siguió la terapia hasta el final. El 25,73\% de los hombres con 2 o más disfunciones sexuales refirió utilizar algún tipo de inhibidores de fosfodiesterasa-5 por más de 10 años; siendo mayor en los menores de 40 años, los cuales los consumen casi cuatro veces más (OR: 3,89; IC95\%; 1,57-10,39; $\mathrm{p} \leq 0,001$ ) respecto de los mayores de 40 años.

El análisis por edades, de la población de hombres con disfunción sexual, reporta una prevalencia global de disfunciones sexuales del 2,66\% en los menores de 30 años, frente al 48,38\% en los mayores de 50 años. Los menores de 30 años mostraron una prevalencia de $1,33 \%$ de trastornos del deseo; con una mediana de 1 disfunción sexual por hombre. En los mayores de 50 años la prevalencia observada fue de $23,65 \%$ de trastornos del deseo y $45,16 \%$ dificultades con la erección; con una mediana de 3 disfunciones sexuales por hombre. En la puntuación del MGH-SFQ se encuentran menores valores en los hombres mayores de 50 años, tanto en los ítems como en el puntaje final del MGH-SFQ $(16,98 \pm 0,90$ versus $19,34 \pm 0,98$, respectivamente), así como en los puntajes de cada dominio (-Tabla 4).

La ansiedad / depresión son un factor de riesgo independiente para deseo sexual hipoactivo en hombres (OR: 1,88; IC95\%: 1,53-2,76). Se observó una tendencia a incrementar el riesgo ante el aumento del número de patologías crónicas. En relación a los que no presentaban ninguna enfermedad, fue de $46,53 \%$ más para los que padecían entre 1 y 6 enfermedades crónicas (OR: 1,46; IC95\%: $1,01-2,43)$ y más del doble para los que padecían más de 6 enfermedades crónicas (OR: 2,46, IC95\%: 1,26-5,13).

Se observó $37,46 \%$ más de riesgo de deseo sexual hipoactivo para los que se quejaron de exceso de trabajo (OR: 1,73; IC95\%: 1,03-1,96) y casi el triple para aquellos con baja autoestima (OR: 2,63; IC95\%: 1,25-5,79) o ansiedad de desempeño (OR: 2,95; IC95\%: 2,26-3,78). Los conflictos de pareja (OR: 10,51; IC95\%: 3,31-30-31), más de 10 años de convivencia (OR: 2,72; IC95\%: 1,28-6,97), así como la falta de

Tabla 4 Massachusetts General Hospital-Sexual Functioning Questionnaire (MGH-SFQ), por edades, en hombres del Quindío, 2019

\begin{tabular}{|l|l|l|l|l|l|l|l|}
\hline $\begin{array}{l}\text { Rango de } \\
\text { edad }\end{array}$ & $\begin{array}{l}\text { Interés } \\
\text { sexual }\end{array}$ & $\begin{array}{l}\text { Excitación } \\
\text { sexual }\end{array}$ & Orgasmo & Erección & $\begin{array}{l}\text { Satisfacción } \\
\text { sexual global }\end{array}$ & $\begin{array}{l}\text { Puntuación } \\
\text { total }\end{array}$ & $\begin{array}{l}\text { Disfunción } \\
\text { sexual }(\%)\end{array}$ \\
\hline $18-29$ & $3,84 \pm 0,99$ & $3,86 \pm 1,03$ & $3,96 \pm 0,96$ & $3,89 \pm 0,93$ & $3,79 \pm 0,97$ & $19,34 \pm 0,98$ & 6,66 \\
\hline $30-39$ & $3,79 \pm 1,02$ & $3,83 \pm 1,11$ & $3,88 \pm 0,95$ & $3,71 \pm 0,88$ & $3,72 \pm 0,91$ & $18,93 \pm 0,98$ & 9,52 \\
\hline $40-49$ & $3,65 \pm 0,91$ & $3,73 \pm 0,98$ & $3,69 \pm 0,92$ & $3,62 \pm 0,97$ & $3,70 \pm 0,86$ & $18,39 \pm 0,93$ & 21,42 \\
\hline $50-59$ & $3,51 \pm 0,89$ & $3,45 \pm 0,94$ & $3,53 \pm 0,89$ & $3,13 \pm 0,92$ & $3,36 \pm 0,84$ & $16,98 \pm 0,90$ & 36,36 \\
\hline $60-69$ & $3,47 \pm 0,85$ & $3,25 \pm 0,87$ & $3,41 \pm 0,88$ & $3,05 \pm 0,91$ & $3,24 \pm 0,85$ & $16,42 \pm 0,87$ & 41,66 \\
\hline$>70$ & $3,36 \pm 0,83$ & $3,12 \pm 0,92$ & $3,27 \pm 0,86$ & $2,87 \pm 0,95$ & $3,11 \pm 0,84$ & $15,73 \pm 0,88$ & 58,33 \\
\hline
\end{tabular}


higiene en la pareja (OR: 1,53; IC95\%: 1,29-13,51) estuvieron asociados a deseo sexual hipoactivo, mientras que conocer el pasado sexual de la pareja aumentó dos veces el riesgo (OR: 2,27; IC95\%: $1,36-3,98 ; p=0,003$ ).

Los hombres mayores de 50 años, tienen 12 veces más probabilidad de padecer deseo sexual hipoactivo que los menores de 50 años (OR: 12,31; IC95\%: 6,68-23,21). El cáncer reporto una probabilidad casi seis veces mayor (OR: 5,71; IC95\%: 1,48-22,81). El cansancio / estrés incrementa el riesgo casi en tres veces (OR: 2,97: IC95\%: 2,13-4,27), con un aumento de hasta ocho veces en los que padecen trastornos del sueño (OR: 8,48; IC95\%: 6,25-9,73). El bajo desempeño sexual de la pareja aumenta el deseo sexual hipoactivo en 197\% (OR: 2,97; IC95\%: 1,25-7,13; $p=0,001$ ), respecto de aquellas parejas que tienen mejor desempeño sexual.

La prevalencia de deseo sexual hipoactivo, fue casi tres veces mayor en los hombres con antecedente de abuso sexual, frente a los que no tenían dicho antecedente (OR: 2,87; IC95\%: 1,27-5,74; $p=0,009$ ); y se incrementó casi ocho veces en presencia del antecedente de disfunciones sexuales (OR: 7,82; IC95\%: 5,54-8,97). La testosterona baja (testosterona total $<300 \mathrm{ng} / \mathrm{dL}$ ) incrementó $87 \%$ el riesgo de deseo sexual hipoactivo (OR: 1,87; IC95\%: 1,29-2,77).

Los hombres que consumían vitamina $\mathrm{D}$ (2000 UI / diarias) tuvieron una reducción del $36 \%$ en el riesgo de padecer deseo sexual hipoactivo (OR: 0,64; IC95\%: 0,41-0,95). Se encontró como factor protector el consumo de Inhibidores de fosfodiesterasa-5 (OR: 0,73; IC95\%: 0,62-0,91). El riesgo disminuyó con el uso de juguetes sexuales (OR: 0,79; IC95\%: 0,72-0,96; $p=0,021$ ).

No se encontró asociación significativa entre el riesgo de deseo sexual hipoactivo en hombres y alteración de la imagen corporal (auto concepto) (OR: 1,48; IC95\%: 0,54-2,63) o la monotonía (OR: 1,00, IC 95\%: 0,99-1,00). Tampoco se encontró relación con la obesidad (OR: 1,18 ; IC 95\%: $0,48-2,37$ ) o la vasectomía (OR: 1,00, IC95\%: 0,96-1,08).

En el análisis bivariado se observó que los principales factores asociados a deseo sexual hipoactivo, en hombres del Quindío, fueron: edad mayor a 50 años (OR: 12,31; IC95\%: 6,68-23,21), conflictos de pareja (OR: 10,51; IC95\%: 3,31-30,31), trastornos del sueño (OR: 8,48; IC95\%: 6,25-9,73), antecedente de disfunciones sexuales (OR: 7,82; IC95\%: 5,54-8,97) y cáncer (OR: 5,71; IC95\%: 1,48-22,81) (-Tabla 5). Mientras que variables como mantener la armonía en la pareja (OR: 0,61; IC95\%: 0,79) y la expresión de sentimientos a la pareja (OR: 0,68; IC95\%: $0,46-0,95)$ se muestran como factores protectores.

En el análisis multivariado (regresión logística), el riesgo de deseo sexual hipoactivo en hombres del Quindío, aumentó con la edad ( $p=0,0015$; OR: 3,43; IC95\%: 1,27-9,26), convivencia en pareja mayor a 10 años $(p<0,001$; OR: 5,18; IC95\%: 2,77-11,75). La ansiedad de desempeño resultó asociada, de forma significativa, al desarrollo de deseo sexual hipoactivo (OR: 4,63; IC 95\% 1,94-10,56). En la - Tabla 6 se observa con mayor detalle.

El análisis multivariado (regresión logística) muestra una asociación significativa entre el riesgo de padecer deseo sexual hipoactivo, en hombres del Quindío, y tener
Tabla 5 Modelo de regresión logística. Factores asociados al deseo sexual hipoactivo en hombres del Quindío, $2019(n=171)$

\begin{tabular}{|c|c|c|c|c|}
\hline & & IC 95\% & & \\
\hline Factores asociados & OR & inferior & Superior & $\mathrm{p}$ \\
\hline Abuso sexual en la infancia & 2,87 & 1,27 & 5,74 & 0,009 \\
\hline $\begin{array}{l}\text { Adicción a sustancias } \\
\text { psicoactivas }\end{array}$ & 4,31 & 3,04 & 6,13 & 0,000 \\
\hline Ansiedad / depresión & 1,88 & 1,53 & 2,76 & 0,000 \\
\hline Ansiedad de desempeño & 2,95 & 2,26 & 3,78 & 0,000 \\
\hline $\begin{array}{l}\text { Antecedente de disfunción } \\
\text { sexual }\end{array}$ & 7,82 & 5,54 & 8,97 & 0,001 \\
\hline Baja autoestima & 2,63 & 1,25 & 5,79 & 0,000 \\
\hline $\begin{array}{l}\text { Bajo desempeño sexual de } \\
\text { la pareja }\end{array}$ & 2,97 & 1,25 & 7,13 & 0,001 \\
\hline Cáncer & 5,71 & 1,48 & 22,81 & 0,000 \\
\hline Cansancio / estrés & 2,97 & 2,13 & 4,27 & 0,000 \\
\hline Comunicación respetuosa & 0,65 & 0,52 & 0,92 & 0,003 \\
\hline Conflictos de pareja & 10,51 & 3,31 & 30,31 & 0,001 \\
\hline $\begin{array}{l}\text { Conocer el pasado sexual } \\
\text { de la pareja }\end{array}$ & 2,27 & 1,36 & 3,98 & 0,003 \\
\hline Consumo de alcohol & 3,29 & 2,07 & 5,19 & 0,000 \\
\hline $\begin{array}{l}\text { Consumo de vitamina D } \\
\text { (2000 UI / diarias) }\end{array}$ & 0,64 & 0,42 & 0,96 & 0,021 \\
\hline $\begin{array}{l}\text { Convivencia en pareja } \\
\text { mayor a } 10 \text { años }\end{array}$ & 2,72 & 1,28 & 6,97 & 0,000 \\
\hline Edad mayor a 50 años & 12,31 & 6,68 & 23,21 & 0,001 \\
\hline Enfermedades crónicas & 1,59 & 1,01 & 2,43 & 0,003 \\
\hline Exceso de trabajo & 1,73 & 1,03 & 1,96 & 0,003 \\
\hline $\begin{array}{l}\text { Expresión de sentimientos } \\
\text { a la pareja }\end{array}$ & 0,68 & 0,46 & 0,95 & 0,027 \\
\hline $\begin{array}{l}\text { Falta de higiene } \\
\text { en la pareja }\end{array}$ & 1,53 & 1,29 & 13,51 & 0,000 \\
\hline Hacer actividades juntos & 0,44 & 0,34 & 0,68 & 0,042 \\
\hline $\begin{array}{l}\text { Imagen corporal - auto } \\
\text { concepto }\end{array}$ & 1,48 & 0,54 & 2,63 & 0,003 \\
\hline Infidelidad en la pareja & 2,13 & 1,64 & 2,83 & 0,000 \\
\hline $\begin{array}{l}\text { Ingesta de Inhibidores } \\
\text { de fosfodiesterasa-5 }\end{array}$ & 0,78 & 0,63 & 0,93 & 0,024 \\
\hline $\begin{array}{l}\text { Ingesta de } \\
\text { medicamentos }\end{array}$ & 4,27 & 3,05 & 5,27 & 0,001 \\
\hline $\begin{array}{l}\text { Mantener la armonía } \\
\text { en la pareja }\end{array}$ & 0,61 & 0,47 & 0,79 & 0,015 \\
\hline $\begin{array}{l}\text { Medicación con } \\
\text { antidepresivos y/o } \\
\text { ansiolíticos }\end{array}$ & 3,65 & 2,49 & 4,87 & 0,000 \\
\hline Monotonía & 1,00 & 0,99 & 1,00 & 0,003 \\
\hline Obesidad & 1,18 & 0,48 & 2,37 & 0,003 \\
\hline $\begin{array}{l}\text { Respeto a ser personas } \\
\text { diferentes }\end{array}$ & 0,53 & 0,41 & 0,71 & 0,027 \\
\hline Uso de juguetes sexuales & 0,79 & 0,72 & 0,96 & 0,021 \\
\hline Tabaquismo & 2,95 & 1,59 & 5,78 & 0,003 \\
\hline $\begin{array}{l}\text { Testosterona baja } \\
(<300 \mathrm{ng} / \mathrm{dL})\end{array}$ & 1,87 & 1,29 & 2,77 & 0,000 \\
\hline Trastornos del sueño & 8,48 & 6,25 & 9,73 & 0,001 \\
\hline Vasectomía & 1,00 & 0,96 & 1,08 & 0,000 \\
\hline
\end{tabular}


Tabla 6 Análisis multivariado (regresión logística) de posibles factores asociados al deseo sexual hipoactivo en hombres del Quindío, 2019

\begin{tabular}{|l|l|l|l|}
\hline Variable & Valor de $\mathbf{p}$ & OR & IC 95\% \\
\hline Ansiedad / depresión & $<0,001$ & 5,53 & $1,72-18,43$ \\
\hline $\begin{array}{l}\text { Ansiedad de } \\
\text { desempeño }\end{array}$ & 0,0018 & 4,62 & $1,95-10,56$ \\
\hline $\begin{array}{l}\text { Antecedente de } \\
\text { disfunciones sexuales }\end{array}$ & 0,0021 & 1,47 & $1,03-2,22$ \\
\hline Cansancio / estrés & 0,0021 & 2,58 & $1,08-3,28$ \\
\hline Conflictos de pareja & 0,0021 & 1,53 & $1,02-2,37$ \\
\hline $\begin{array}{l}\text { Convivencia en pareja } \\
\text { mayor a 10 años }\end{array}$ & $<0,001$ & 5,19 & $2,71-11,71$ \\
\hline Incremento de la edad & 0,0015 & 3,42 & $1,26-9,36$ \\
\hline Testosterona baja & $<0,001$ & 5,59 & $1,82-18,37$ \\
\hline Trastornos del sueño & 0,0021 & 1,89 & $1,35-2,58$ \\
\hline
\end{tabular}

conflictos de pareja (OR: 1,52; IC95\%: 1,03-2,36), presentar disfunciones sexuales (OR: 1,48; IC95\%: 0,97-2,27) y presentar cansancio / estrés (OR: 1,58; IC95\%: 1,07-2,29) (-Tabla 6).

El déficit de testosterona se incorporó en el análisis multivariado (regresión logística), como significativamente asociado a la aparición de deseo sexual hipoactivo en hombres del Quindío (OR: 5,59; IC95\%: 1,84-18,53), de igual manera se objetivó la ansiedad / depresión (OR: 5,56; IC95\%: 1,73-18,83) y los trastornos del sueño (OR: 1,87; IC95\%: 1,38-2,59) (-Tabla 6).

La comparación entre las enfermedades crónicas permitió establecer diferencias entre las patologías analizadas, se obtuvo un resultado significativo en el análisis multivariado, para el antecedente de padecer disminución de la testosterona, hipotiroidismo, diabetes, dislipidemia y cáncer (OR ajustada: 3,13; IC 95\%: 1,37 - 7,36).

\section{Discusión}

La especie humana se caracteriza por ser sexual, sexuada y erótica; donde la motivación del deseo sexual la constituyen dos valiosos elementos: atracción y enamoramiento. ${ }^{10,14} \mathrm{La}$ falta de deseo sexual, en las últimas décadas, dejó de ser un trastorno propio de las mujeres. Si en el pasado eran ellas las que consultaban, hoy son ellos los que más buscan ayuda por la misma dificultad. ${ }^{14}$

Las alteraciones del deseo sexual se ven inducidas por procesos que suelen ser multifactoriales, por lo tanto, en su desarrollo contribuyen factores tanto biológicos (hormonal, neurológico, orgánico, vascular) como psicológicos y socioculturales. Entre los factores biológicos se incluyen enfermedades, terapias empleadas (quirúrgicas o médicas). En los factores psicológicos se citan problemas de autoestima, ansiedad, depresión, etc. ${ }^{9,10,14}$

A la luz de los resultados de esta investigación, la magnitud del problema de las disfunciones sexuales en hombres del Quindío, donde más de 1/5 de la población presenta alguna disfunción sexual, con un 21,63\% de trastornos con la erección y 15,78\% de afectación del interés sexual (incrementándose a más del doble a partir de los 50 años); podemos observar que la prevalencia de disfunciones sexuales es significativamente inferior al $85,81 \%$ descrito por Espitia y col., ${ }^{11}$ en hombres del Quindío, para la población de mayores de 50 años; al 53,4\% presentado en el estudio Disfunción Eréctil en el Norte de Sudamérica (DENSA) ${ }^{15}$; eso puede atribuirse tanto al tipo de instrumento utilizado por nosotros, como a lo heterogéneo de la población con relación a la edad de los participantes.

En relación a la prevalencia de deseo sexual hipoactivo en hombres del Quindío, nuestros hallazgos son concordantes con investigaciones publicadas por Frank y col., en Inglaterra quienes encontraron una frecuencia de $16 \%$ de los esposos con falta de interés en el sexo. ${ }^{16}$ Laumann y col., en 1.410 hombres estadounidenses, de 18 a 59 años, reportan una prevalencia del $15 \%$ de hombres que carecieron de interés sexual durante varios meses en el último año ${ }^{17}$; Lindau y col., informan una prevalencia del $28 \%$ de falta de interés en el sexo, ${ }^{18}$ con un $65 \%$ de molestia asociada al síntoma; ese último dato es mucho más alto porque se trató de una población de hombres, exclusivamente mayores de 55 años y con variables diferentes.

El angustioso deseo sexual hipoactivo puede atribuirse a una serie de alteraciones biológicas, psicológicas, sociales y contextuales o de pareja. ${ }^{9,10,14}$ En concordancia, nosotros informamos la clara influencia de la edad mayor a 50 años (OR: 12,31; IC95\%: 6,68-23,21), conflictos de pareja (OR: 10,51; IC95\% 3,31-30,31), trastornos del sueño (OR: 8,48; IC95\%: 6,25-9,73), antecedente de disfunción sexual (OR: 7,82; IC95\%: 5,54-8,97), cáncer (OR: 5,66; IC95\%: 1,48-22,81), adicción a sustancias psicoactivas (OR: 4,31; IC95\%: 3,04-6,13), etc., lo cual se alinea con el componente multifactorial descrito por Goldstein y col., en el año 2017, en el consenso de expertos de la Sociedad Internacional para el Estudio de la Salud Sexual de las Mujeres ${ }^{19}$; lo cual es totalmente aplicable a la población de hombres del Quindío.

En el estudio de Cabada-Ramos y col., reportan que un $84,4 \%$ de los hombres experimentaron cambios en su función sexual debido a alguna enfermedad y al efecto secundario de los medicamentos administrados para tratarla ${ }^{20}$; CrespoIglesias y col., en el 2006 (España), en una población de pacientes psiquiátricos señalan un $87,9 \%^{21}$; por su parte Agle y col., (E.E. U.U), encontraron un $82 \%{ }^{22}$ Resultados que dejan claro como las enfermedades crónicas, no solo alteran al individuo por el proceso de adaptación que implican en sí mismas, sino que pueden generar dificultades económicas, cambios en la visión que la persona tiene de sí misma, y afectar las relaciones con los miembros de la familia, amigos y el entorno socio-cultural.

Rowe y col., definen el envejecimiento exitoso como tener una baja probabilidad de enfermar y discapacidad asociada a ella, un alto funcionamiento a nivel físico y cognitivo y un compromiso activo con la vida ${ }^{23}$; de esa manera, la sexualidad en la vida de las personas mayores, hace parte del envejecimiento exitoso; teniendo en consideración su dimensión biológica, mental, social y espiritual. Es que el 
sentimiento de estar sexualmente satisfecho, implica tener una integración adecuada de todas dichas esferas ${ }^{24}$; lo cual resulta en contraposición a nuestros resultados, con un OR: 12,31 (IC95\%: 6,68-23,21), concluyendo que ser mayor de 50 años, es un factor asociado a deseo sexual hipoactivo. Estableciéndose la realidad de que en el momento en que la función sexual se convierte en un problema de cualquier tipo, es fuente de angustia y frustración, generando una sensación de malestar e insatisfacción en las relaciones interpersonales. $^{5}$

En hombres con depresión, entre el 50-70\%, presentan trastornos de la esfera sexual, ${ }^{25,26}$ los que se agravan con la terapia antidepresiva, ${ }^{27}$ por lo tanto, tienen un factor de riesgo adicional, como lo es el consumo de antidepresivos (OR: 3,66; IC95\%: 2,49-4,89); sin embargo, a pesar de la creciente prevalencia de bajo deseo sexual en los hombres, se sabe poco sobre las características y el impacto de ese trastorno en la población de hombres en Colombia. ${ }^{10,14}$

Al analizar la infidelidad, Lawson y col., reportan que la prevalencia de las aventuras parece estar aumentando entre las mujeres, especialmente entre las más jóvenes, las cuales están participando en aventuras, en un porcentaje más elevado que sus esposos. ${ }^{28}$ En nuestro estudio se reportó un $10,52 \%$ de infidelidad femenina, duplicando el riesgo de deseo sexual hipoactivo en hombres (OR: 2,13; IC95\%: $1,64-2,83)$, lo que ratifica las consecuencias devastadoras de la infidelidad en las relaciones de pareja.

Pinto y col., refiere que a pesar de que ambos miembros se involucren en la relación, cuando no encuentran la manera más adecuada de resolver los conflictos, la relación puede terminar. ${ }^{29}$ Nosotros encontramos que los conflictos de pareja, con un OR: 10,51 (IC95\%: 3,31-30,31), se constituyeron en un significativo factor asociado al deseo sexual hipoactivo.

En fin, los hombres hacen parte de una nueva sociedad, donde la cultura les exige y demanda autonomía, estabilidad (afectiva, económica y emocional), perfección, productividad y valor; por lo tanto, se encuentran rodeados de un entorno prolijo en estresores que impactan de a poco, pero de forma constante, en el desempeño y funcionamiento sexual.

Ser hombre en la actualidad representa tener que vivir en un grupo familiar que debe defender, proteger y sostener, donde muchas veces encuentra un pobre soporte emocional, atiborrado de una constelación de problemas de comunicación (continuas discusiones, críticas, juicios, etc.). En suma, es un conjunto que puede actuar de forma negativa, empobreciendo no solo su autoimagen, sino su eficiencia y desempeño en la esfera sexual.

Los hallazgos de nuestro estudio, dejan al descubierto, la necesidad de implementar programas encaminados a la evaluación de las disfunciones sexuales en hombres, lo cual le permite a esa población buscar la ayuda necesaria, a fin de resolver sus eventuales conflictos sexuales.

En las fortalezas de esta investigación destacamos el muestreo aleatorio, lo que no restringe la representatividad de la población. Otra fortaleza la constituye el número de hombres participantes en el estudio. Así, la estimación de la prevalencia y de los factores asociados, no se ven afectadas por el bajo número de integrantes, lo cual se refleja en los intervalos de confianza de las estimaciones del deseo sexual hipoactivo y en los factores asociados, los que se amplían por ejemplo en el caso del abuso sexual en la infancia o el uso de juguetes sexuales. Son obvias las limitaciones inherentes a un estudio transversal, dado que estudiar en simultánea los factores asociados al deseo sexual hipoactivo (en una población definida de hombres), en un momento determinado, no permite establecer la secuencia temporal de los sucesos ni determinar si la exposición precedió al trastorno o viceversa. Se pudo haber subestimado la prevalencia de deseo sexual hipoactivo en hombres del Quindío, debido a que el estudio no incluye población fuera de la clínica. El uso del MGH-SFQ en la evaluación del funcionamiento sexual de los hombres, a pesar de tener excelentes propiedades psicométricas, es un cuestionario auto administrado, y eso puede generar sesgos.

Conflicto de Intereses

Los autores declaran no tener ningún conflicto de intereses.

\section{Agradecimientos}

Nuestro sincero agradecimiento a las directivas de Hathor, Clínica Sexológica; por ayudarnos a gestionar los permisos y protocolos para poder desarrollar esta investigación, así como a los hombres participantes, sin cuya valiosa colaboración y participación, no hubiese sido posible realizar el estudio.

\section{Referencias}

1 Rosen RC. Prevalence and risk factors of sexual dysfunction in men and women. Curr Psychiatry Rep 2000;2(03):189-195. Doi: 10.1007/s11920-996-0006-2

2 Cohen SD. The challenge of erectile dysfunction management in the young man. Curr Urol Rep 2015;16(12):84

3 Espitia-De La Hoz FJ. Prevalencia de disfunción eréctil en hombres del Quindío, y factores de riesgo asociados. Urol Colomb 2019;28 (02):169-176. Doi: 10.1055/s-0038-1656515

4 Simons JS, Carey MP. Prevalence of sexual dysfunctions: results from a decade of research. Arch Sex Behav 2001;30(02):177-219. Doi: 10.1023/a:1002729318254

5 Mercer $\mathrm{CH}$, Fenton KA, Johnson AM, et al. Sexual function problems and help seeking behaviour in Britain: national probability sample survey. BMJ 2003;327(7412):426-427. Doi: 10.1136/bmj.327.7412.426

6 Bancroft J. Human sexuality and its problems (3rd ed.). London, UK: Elsevier; 2009

7 Kaplan HS. Hypoactive sexual desire. J Sex Marital Ther 1977;3 (01):3-9. Doi: 10.1080/00926237708405343

8 Regan PC, Berscheid E. Lust: What we know about human sexual desire. Thousand Oaks, CA: Sage; 1999

9 Espitia-De La Hoz FJ. Diagnóstico y tratamiento farmacológico del trastorno del deseo sexual hipoacivo femenino. Revista Desexol 2018;7(02):36-52

10 Espitia-De La Hoz FJ. Sexo Ilimitado / El vuelo hacia una sexualidad más placentera. Primera edición. Bogotá. Editorial Bolívar 2015;1(15):52-55

11 Espitia De La Hoz FJ. Prevalence and characterisation of sexual dysfunctions in women, in 12 Colombian cities, 2009-2016. Rev. Colomb. Obstet. Ginecol. 2018;69(01):9-21. Doi: http://dx.doi. org/10.18597/rcog.3035 
12 Labbate LA, Lare SB. Sexual dysfunction in male psychiatric outpatients: validity of the Massachusetts General Hospital Sexual Functioning Questionnaire. Psychother Psychosom 2001; 70(04):221-225

13 Sierra JC, Vallejo-Medina P, Santos-Iglesias P, Lameiras Fernández M. [Validation of Massachusetts General Hospital-Sexual Functioning Questionnaire (MGH-SFQ) in a Spanish population]. Aten Primaria 2012;44(09):516-524

14 Espitia-De La Hoz FJ. Mi primer año en La Crónica. Primera edición. Bogotá. Editorial Bolívar 2016;7(01):87-88

15 Morillo LE, Díaz J, Estevez E, et al. Prevalence of erectile dysfunction in Colombia, Ecuador, and Venezuela: a population-based study (DENSA). Int J Impot Res 2002;14 (Suppl 2):S10-S18. Doi: 10.1038/sj.ijir.3900893

16 Frank E, Anderson C, Rubinstein D. Frequency of sexual dysfunction in "normal" couples. N Engl J Med 1978;299(03): 111-115. Doi: 10.1056/NEJM197807202990302

17 Laumann EO, Paik A, Rosen RC. Sexual dysfunction in the United States: prevalence and predictors. JAMA 1999;281(06):537-544. Doi: $10.1001 /$ jama.281.6.537

18 Lindau ST, Schumm LP, Laumann EO, Levinson W, O'Muircheartaigh CA, Waite LJ. A study of sexuality and health among older adults in the United States. N Engl J Med 2007;357 (08):762-774. Doi: 10.1056/NEJMoa067423

19 Goldstein I, Kim NN, Clayton AH, et al. Hypoactive Sexual Desire Disorder: International Society for the Study of Women's Sexual Health (ISSWSH) Expert Consensus Panel Review. Mayo Clin Proc 2017;92(01):114-128. Doi: 10.1016/j.mayocp.2016.09.018
20 Cabada-Ramos E, Bueno-Osorio I, Casillas VE, et al. Cambios en la función sexual en hombres y mujeres de 40 a 59 años de edad. Rev Esp Med Quir 2011;16(01):8-12

21 Crespo Iglesias JM, Prado Robles V, Pérez García M. Valoración de la función sexual en pacientes psiquiátricos crónicos ingresados en una unidad de rehabilitación psicosocial. Actas Esp Psiquiatr 2006;34(01):41-47

22 Agle DP, Baum GL. Psychological aspects of chronic obstructive pulmonary disease. Med Clin North Am 1977;61(04):749-758

23 Rowe JW, Kahn RL. Successful aging. Gerontologist 1997;37(04): 433-440

24 Barton D, Joubert L. Psychosocial aspects of sexual disorders. Aust Fam Physician 2000;29(06):527-531

25 Angst J. Sexual problems in healthy and depressed persons. Int Clin Psychopharmacol 1998;13(Suppl 6):S1-S4

26 Bonierbale M, Lançon C, Tignol J. The ELIXIR study: evaluation of sexual dysfunction in 4557 depressed patients in France. Curr Med Res Opin 2003;19(02):114-124

27 Montejo-González AL, Llorca G, Izquierdo JA, et al. SSRI-induced sexual dysfunction: fluoxetine, paroxetine, sertraline, and fluvoxamine in a prospective, multicenter, and descriptive clinical study of 344 patients. J Sex Marital Ther 1997;23(03):176-194

28 Lawson A. Adultery: An analysis of love and betrayal. New York: Basic Books; 1988

29 Pinto B. Amor y personalidad en universitarios aymaras del departamento de La Paz. Tesis doctoral, Universidad de GranadaEspaña2008 
Anexo 1 Versión validada en español del Massachusetts General Hospital-Sexual Functioning Questionnaire (MGH-SFQ)

\begin{tabular}{|c|c|c|c|c|c|}
\hline Preguntas & $\begin{array}{l}\text { Totalmente } \\
\text { disminuido }\end{array}$ & $\begin{array}{l}\text { Marcadamente } \\
\text { disminuido }\end{array}$ & $\begin{array}{l}\text { Moderadamente } \\
\text { disminuido }\end{array}$ & $\begin{array}{l}\text { Minimante } \\
\text { disminuido }\end{array}$ & Normal \\
\hline $\begin{array}{l}\text { 1. ¿Cómo ha estado su interés sexual } \\
\text { durante el último mes? }\end{array}$ & 0 & 1 & 2 & 3 & 4 \\
\hline $\begin{array}{l}\text { 2. ¿Cómo ha estado su capacidad } \\
\text { para conseguir excitación sexual } \\
\text { en el último mes? }\end{array}$ & 0 & 1 & 2 & 3 & 4 \\
\hline $\begin{array}{l}\text { 3. ¿Cómo ha estado su capacidad } \\
\text { para alcanzar el orgasmo en el } \\
\text { último mes? }\end{array}$ & 0 & 1 & 2 & 3 & 4 \\
\hline $\begin{array}{l}\text { 4. ¿Cómo ha estado su capacidad para } \\
\text { conseguir y mantener una erección } \\
\text { en el último mes? (Sólo hombres) }\end{array}$ & 0 & 1 & 2 & 3 & 4 \\
\hline $\begin{array}{l}\text { 5. ¿Cómo calificaría su satisfacción sexual } \\
\text { general en el último mes? }\end{array}$ & 0 & 1 & 2 & 3 & 4 \\
\hline Puntaje mínimo & 0 & & & & \\
\hline Puntaje máximo & 20 & & & & \\
\hline
\end{tabular}

